\title{
Regional variability in fecal microbiota transplantation practices: a survey of the Southern Ontario Fecal Microbiota Transplantation Movement
}

\author{
Susy S. Hota MD MSc, Salman Surangiwala, Aimee S. Paterson MSc, Bryan Coburn MD PhD, \\ Susan M. Poutanen MD MPH; for the Southern Ontario Fecal Microbiota Transplantation (SOFT) \\ Movement*
}

\section{Abstract}

Background: There is growing evidence that fecal microbiota transplantation (FMT) is an effective treatment for recurrent Clostridium difficile infection, but little guidance exists for implementation of FMT programs. The objective of this study is to describe the program characteristics and protocols of 9 planned or operating FMT programs in the Southern Ontario Fecal Microbiota Transplantation (SOFT) Movement, to help guide future FMT program implementation.

Methods: A 59-item survey was administered electronically to clinical leads of the SOFT Movement on June 2, 2016. The survey evaluated 7 domains: FMT program characteristics, FMT recipients, donor screening/selection, transplant manufacturing, FMT administration, good manufacturing procedures/biosafety procedures and infection-control procedures. We used descriptive statistics to analyze quantitative data.

Results: All 9 programs responded to the survey: 6 were active, 1 had FMT standard operating procedures developed but did not have clinical experience, and 2 were in the process of forming FMT programs. All 6 active programs performed FMT in adult patients with C. difficile infection. About 1300 FMT procedures were performed between 2003 and 2016. Five of the 6 operating programs administered the preparation via enema. Programs were driven primarily by physicians. All programs used universal FMT donors and followed Health Canada's screening guidelines, with considerable variability in screening frequency (every 3-6 mo) and modality. Locations for transplant preparation and manufacturing protocols varied across programs. Stool mass for FMT ranged from $20 \mathrm{~g}$ to $150 \mathrm{~g}$, and transplant volume ranged from $25 \mathrm{~mL}$ to $300 \mathrm{~mL}$.

Interpretation: The experience of this high-volume regional FMT network highlights current challenges in FMT program development, including a high reliance on physicians and the costly nature of donor screening. Standardization and optimization through development of regional centres of excellence for FMT donor recruitment and administration should be explored.

ver the past decade, fecal microbiota transplantation (FMT) has emerged as a novel treatment option for recurrent Clostridium difficile infection. Multiple randomized controlled trials have shown that FMT using various methodologies is at least as effective as, if not superior to, conventional antibiotic treatments for recurrent C. difficile infection. ${ }^{1-7}$ However, for clinicians and centres considering offering FMT, little to no guidance exists on how to approach program development. In part, this stems from a lack of accepted standards for FMT methodologies. Within FMT research, variables such as patient selection, timing of FMT, pretreatment (with antibiotics and bowel lavage), donor selection, donor screening, manufacturing and storage conditions, route of administration and number of administrations have not been adequately standardized and validated. ${ }^{8,9}$
In 2015, Health Canada issued brief, interim guidance regarding the use of FMT in patients with $C$. difficile infection not responding to conventional treatment, focusing on the safety aspects of donor selection and screening. ${ }^{10}$ Although various expert bodies have produced guidelines for FMT that include further details on how to manufacture the preparation and perform FMT, these recommendations are based

Competing interests: See the end of the article.

*A list of the physician leads is provided at the end of the article.

This article has been peer reviewed.

Correspondence to: Susy Hota, susy.hota@uhn.ca

CMAJ Open 2018. DOI:10.9778/cmajo.20170109 
predominantly on expert opinion. ${ }^{11,12}$ In the absence of evidence-informed guidance, individual centres in Canada offering FMT have largely created their own protocols.

In November 2015, the Southern Ontario Fecal Microbiota Transplantation (SOFT) Movement was created with the dual purpose of sharing FMT experience between longstanding and new programs and removing barriers to FMT provision in Ontario. Physicians known to be performing FMT or planning to perform it in southern Ontario, based on prior communication through the Association of Medical Microbiology and Infectious Disease Canada, were invited to be SOFT Movement members. The objective of this study was to describe the characteristics and protocols of planned or operating FMT programs in the SOFT Movement, to help guide future FMT program implementation.

\section{Methods}

\section{Setting and participants}

The study took place in southern Ontario, a region of 12.8 million people representing nearly one-third of Canada's population. ${ }^{13}$ Twelve SOFT Movement clinical leads representing 9 hospital corporations in southern Ontario (Figure 1) were approached. For institutions with more than 1 clinical lead (University Health Network/Sinai Health System, St. Joseph's Health Centre and Michael Garron Hospital [formerly Toronto East General Hospital], Toronto), participants were instructed to collaboratively complete 1 survey.

\section{Survey development and administration}

A 59-item closed survey (Appendix 1, available at www. cmajopen.ca/content/6/2/E184/suppl/DC1) was developed by 2 study investigators (S.S.H. and S.M.P.) and was reviewed for content validity by a third investigator (B.C.). A fourth investigator (S.S.) tested the survey for usability. Open-ended and multiple-choice questions evaluated 7 domains based on consultation with all SOFT Movement members: 1) FMT program characteristics, 2) recipients, 3) donor screening and selection, 4) transplant manufacturing, 5) transplant administration, 6) good manufacturing procedures/biosafety and 7) infection-control procedures. Items were displayed sequentially, with 4-12 items per screen, over 8 screens. We built the survey using SurveyGizmo and administered it electronically on June 2, 2016.

Participants received an email containing a link to the online survey, with instructions to submit responses within 2 weeks. No incentives were provided. One reminder email was sent. The survey took about 20 minutes to complete.

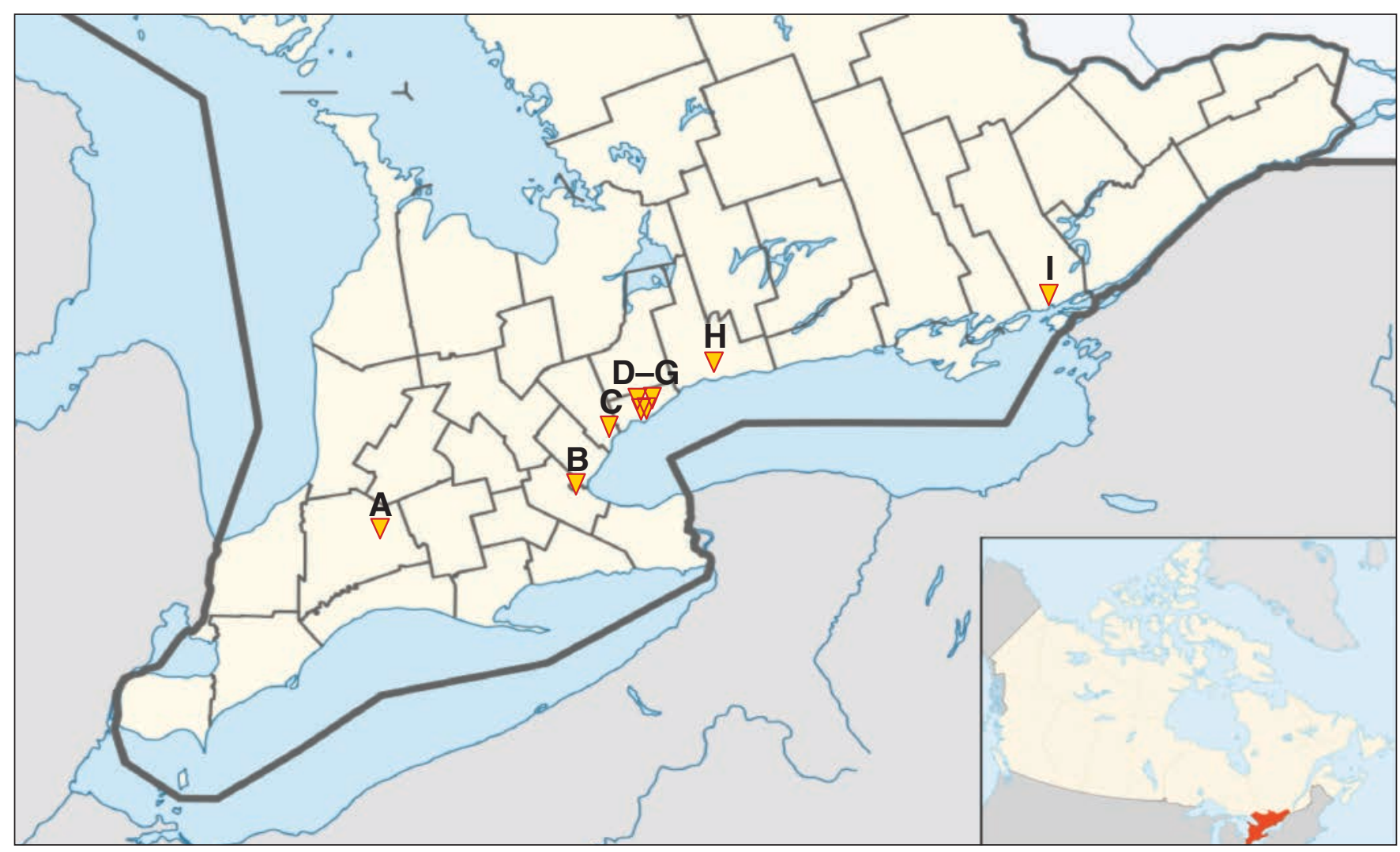

Figure 1: Location of fecal microbiota transplantation (FMT) programs in Southern Ontario Fecal Microbiota Transplantation (SOFT) Movement, 2016. Programs included: A) St. Joseph's Hospital, London, B) St. Joseph's Healthcare, Hamilton, C) Trillium Health Partners, Mississauga, D) University Health Network/Sinai Health System, Toronto, E) Michael Garron Hospital, Toronto, F) St. Joseph's Health Centre, Toronto, G) Sunnybrook Health Science Centre, Toronto, H) Lakeridge Health, Oshawa and I) Kingston General Hospital, Kingston. Sunnybrook Health Sciences Centre and St. Joseph's Health Centre were not operating at the time of the survey. Lakeridge Health had standard operating procedures developed but had not yet performed FMT in a patient. Adapted from Wikimedia Commons User:NordNordWest. 
Respondents were able to review their responses and could return to complete the survey at a later time.

\section{Data management and analysis}

Survey data were entered manually into a Microsoft Excel spreadsheet stored on a secure server at Mount Sinai Hospital. Prevention of duplicate entries was confirmed manually as respondents and institutions were identified on the survey. In cases in which incomplete responses were submitted, 1 repeat contact was made. Incomplete survey data were included in the analysis. We analyzed the data using standard descriptive statistics in Microsoft Excel. There was no weighting of items.

\section{Ethics approval}

Consent to participate in the study was implicit to completing the survey, and the survey participants were aware of the study purpose and data management plan in advance. Institutional research ethics board approval was not deemed necessary as participation was voluntary, the survey did not request patient information or elicit personal opinions, and results were to be presented in aggregate.

\section{Results}

All 9 institutions responded to the survey request, with 6 providing complete responses. One institution that had FMT standard operating procedures developed but did not have clinical experience provided incomplete responses, answering only where applicable to their procedures. Two other institutions were in the process of forming FMT programs and therefore also provided incomplete responses.

\section{Program characteristics}

Since 2003, about 1300 FMT procedures have been performed across 6 institutions. The earliest program began in 2003, and the remainder of the programs started after 2008. One program performed FMT for clinical care only, whereas the remaining programs planned or performed FMT for clinical care and research. All the programs were staffed by physicians. In addition, 5 programs had interprofessional clinical support (program coordinator, nurses and physician assistant), and 2 employed research staff (research nurse and summer student).

\section{Recipients}

All active programs performed FMT in adult patients with C. difficile infection. One centre additionally performed research into FMT for inflammatory bowel disease, another was researching FMT for nonalcoholic fatty liver disease and multiple sclerosis, and a third was planning research on FMT for obesity and bipolar depression. Indications for FMT used by the programs for patients with $C$. difficile infection are summarized in Figure 2. Major exclusion criteria for FMT recipients included severe, uncontrollable diarrhea (3 programs), bloody diarrhea (2), any immunocompromise (1),

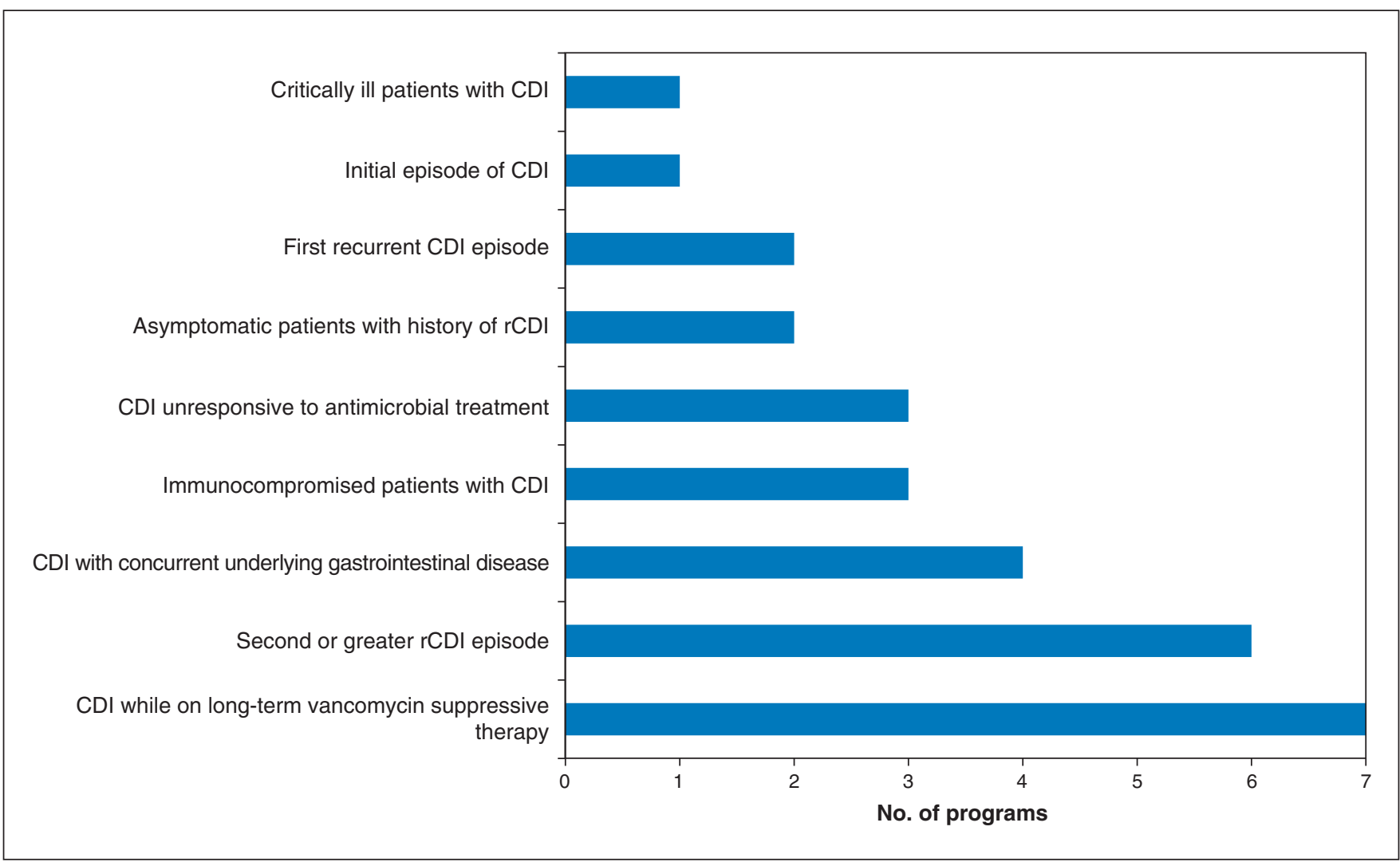

Figure 2: Number of operating or planned programs that reported providing fecal microbiota transplantation to subgroups of patients with Clostridium difficile infection (CDI) or recurrent CDI (rCDI). 
neutropenia (1) and irreversible bleeding disorder (1). No program had an upper age limit for FMT recipients.

\section{Donor screening and selection}

All programs used or planned to use universal donors. At all sites, universal donors underwent screening at baseline. Five of 7 institutions screened or planned to screen universal donors every 6 months thereafter, 1 institution screened every 3 months, and 1 institution screened every 3 months and at the end of the donation cycle. Three programs performed microbiota analysis of the donor feces, but none used these data as selection criteria for donors.

All programs excluded donors with the noninfectious disease exposures or agents recommended by Health Canada, ${ }^{10}$ except that 1 program allowed magnesium consumption. All programs excluded donors with a history of cancer, and some excluded those with chronic medical conditions, those with ongoing use of medications and those consuming certain foods, depending on recipients' allergies.

Figure 3 summarizes the microorganisms and diseases that the programs reported screening for to adhere to Health Canada's recommended donor exclusion criteria. ${ }^{10}$ All tests are performed on stool samples with the exception of HIV-1, HIV-2, human T-cell lymphotropic virus type I and II, hepatitis B and C, syphilis and Helicobacter pylori (serum samples), and Chlamydia trachomatis and Neisseria gonorrboeae (urine and stool samples). A substantial proportion of infectious disease screening was done at reference laboratories. A history of gastroenteritis (diarrhea) due to agents not directly screened for in laboratory tests (e.g., Listeria), malaria, Chagas disease, babesiosis, or Creutzfeldt-

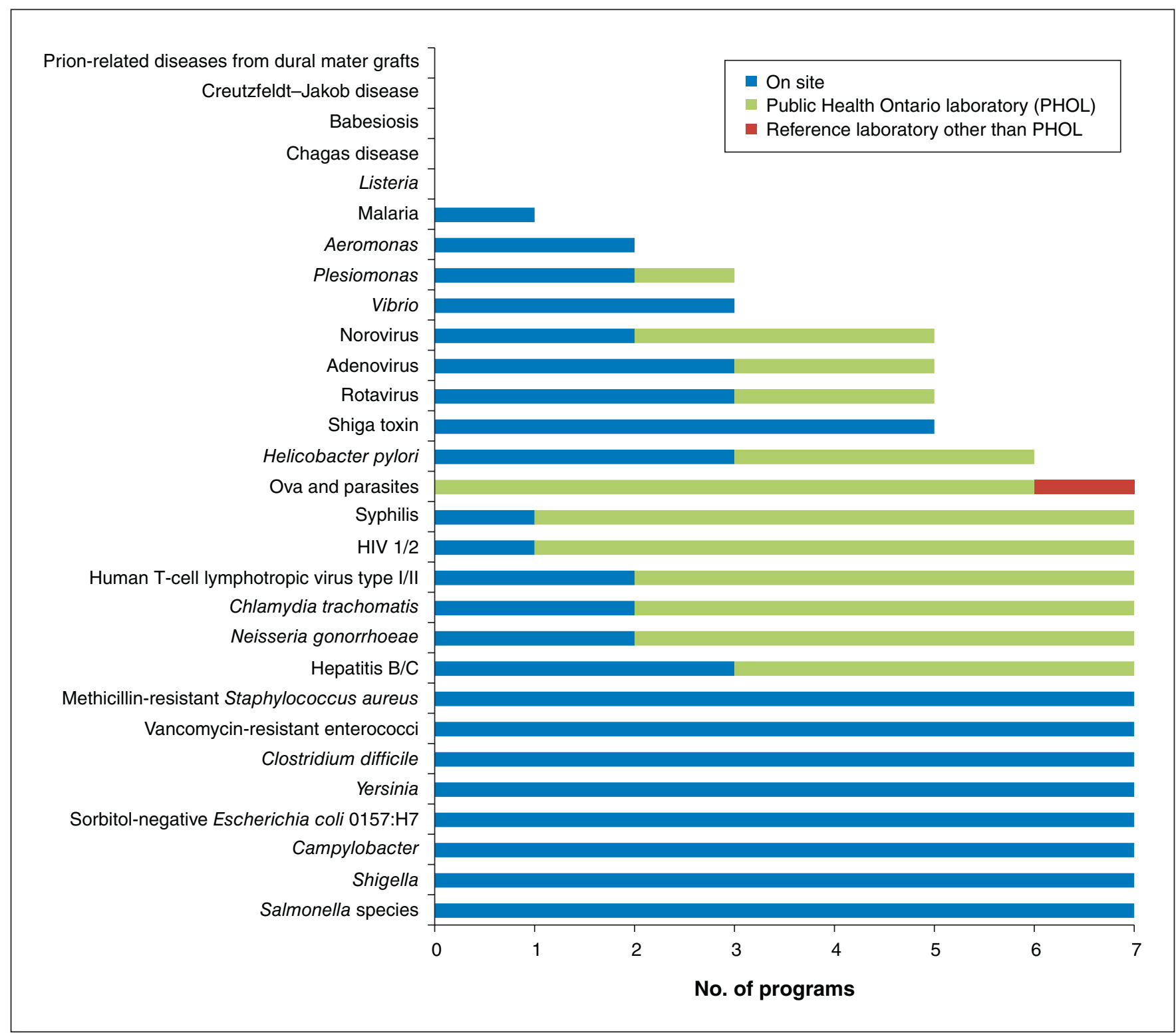

Figure 3: Number of operating or planned programs that reported completing laboratory testing for the infectious disease agents and diseases recommended by Health Canada. ${ }^{10}$ 
Jakob disease or other prion disease was determined from medical assessment in all cases, with 1 centre also performing laboratory testing for malaria. Although not specifically recommended by Health Canada, 5 programs screened for carbapenemaseproducing Enterobacteriaceae, and 4 screened for extendedspectrum $\beta$-lactamase-producing organisms.

\section{Manufacturing}

Three of the 6 active programs manufactured the preparation in a clinical microbiology laboratory, 2 manufactured it in a research laboratory, and 1 used a shared clinical/research space. In 3 programs, a physician prepared the transplant all or some of the time; in other programs, transplant manufacturing involved a nurse (1 program), laboratory technologists (1), research technologist (1), PhD microbiologist (1) or physician assistant (1).

Four programs carried out manufacturing validation studies before finalizing protocols confirming that their manufacturing procedures did not compromise the diversity of microbiota. Four programs recommended that donor feces be stored in household refrigerators before being transported to the FMT centre. Most programs strived to prepare the transplant within 24 hours of donation, although 2 centres accepted samples up to 48 and 72 hours after donation.

Programs reported a wide range of donor stool mass and diluent volume in manufacturing the preparation (Table 1). Diluents varied, with 3 programs using saline, 1 using water, and 2 using saline for FMT administered by enema and water for FMT administered by nasojejunal tube. Six programs manufactured frozen transplants, and 1 institution additionally offering lyophilized transplants. Only 1 program used a cryoprotectant (glycerol) in the frozen product. When frozen, the preparation was maintained at both $-20^{\circ} \mathrm{C}$ and $-80^{\circ} \mathrm{C}$.

\section{Administration}

The most common route of administration was enema: 3 programs offered FMT by enema alone, 1 provided enema and colonoscopy administration, and 1 provided enema and nasojejunal administration. One institution performed FMT by the nasojejunal route only.

Fecal microbiota transplantation was performed in a clinic, inpatient room or day unit. It was most commonly administered by a physician ( 5 programs), sometimes with assistance from a physician trainee, physician assistant or nurse. In most cases, when FMT was being administered to patients with C. difficile infection, oral vancomycin therapy was stopped

\begin{tabular}{|c|c|c|}
\hline $\begin{array}{l}\text { Route of } \\
\text { administration }\end{array}$ & $\begin{array}{l}\text { Range of mass of } \\
\text { stool used, } g\end{array}$ & $\begin{array}{l}\text { Range of diluent } \\
\text { used, } \mathrm{mL}\end{array}$ \\
\hline Enema & $20-100$ & $25-300$ \\
\hline Colonoscopy & $30-150$ & $200-300$ \\
\hline Nasojejunal & $30-100$ & $70-250$ \\
\hline
\end{tabular}

24-48 hours before FMT, although 1 site stopped it 48-96 hours before and another, less than 24 hours before. Only 1 program used a bowel preparation before FMT. In the centres that administered FMT by enema, 2 programs administered FMT once, 3 provided it up to 3 times, and 1 provided more than 5 transplants if necessary. In the 2 programs providing or planning to provide FMT by colonoscopy, a single administration was/would be given. One program provided a single transplant via a nasojejunal tube, and another provided up to 3 nasojejunal administrations. When multiple transplants were administered, they were most frequently given 2-4 days apart.

At 3 sites, patients were not routinely followed after FMT, whereas 4 sites reported variable follow-up durations, ranging from 1 to 36 months.

\section{Good manufacturing procedures/biosafety procedures}

All programs manufactured the transplant in a biosafety cabinet. Two programs used disposable equipment for manufacturing, and the remainder disinfected the manufacturing equipment and space using a sporicidal agent.

\section{Infection-control procedures}

Fluid-resistant gowns and gloves were used by all programs during transplant manufacturing and administration; procedure masks and face shields were used variably. Shoe covers were used during FMT at 1 institution. Four programs performed more than 1 FMT per day. Between cases, 2 of these programs requested environmental services to disinfect rooms with a sporicidal agent, 1 relied on FMT program staff do this, and 1 only changed linens between cases. All programs requested environmental services to disinfect rooms used for FMT with a sporicidal agent after the last procedure of the day.

\section{Interpretation}

Collectively, our FMT programs have performed about 1300 procedures, primarily in patients with recurrent $C$. difficile infection, but programs are expanding to include other research indications. The preparation is most commonly administered by enema, although colonoscopic and nasojejunal routes are also available. Biotherapeutics (encapsulated stool-derived organisms) were not available in the region at the time of our study. The programs are heavily driven by physicians. Universal FMT donors are widely used, primarily to provide feces that are frozen and banked for later use. Transplant manufacturing is highly variable across programs. The total weight of stool used, the volume of diluent and the method of preparation differs across sites, and manufacturing often occurs in research laboratories.

To date, no definitive data support one route of administration (enema, colonoscopy or nasojejunal tube) over another regarding FMT efficacy for recurrent $C$. difficile infection. ${ }^{3,714-18}$ Administration via enema or nasojejunal tube may be performed by a nurse or physician assistant, but a physician with expertise in gastroenterology, infectious diseases and/or 
microbiology is required to oversee the procedure, donor screening and recipient follow-up. This introduces a dependency on physicians for FMT program functionality. As there is currently no mechanism for compensating physicians for FMT in Ontario, physicians are donating time and expertise to provide this service. Hospitals are also absorbing costs associated with building and supporting FMT programs, most notably including donor screening. Universal FMT donors are cost-effective and validated ${ }^{4,19-21}$ and facilitate providing FMT on demand. The optimal frequency for screening universal donors is not clear, and variability exists among our programs. Comprehensive donor screening, as recommended by Health Canada, ${ }^{10}$ is expensive, and not all tests are readily available everywhere. There is a heavy reliance on reference laboratories for specialized testing in our programs. In some cases, validated or reliable tests do not exist (e.g., testing of stool for Listeria), yet medical assessment alone may not necessarily exclude the possibility of infection or carriage. Transplant manufacturing presents further challenges. Locating a space for this task is a frequent problem, resulting in physicians' using research space to manufacture a clinical therapeutic. Currently, there are no evidence-based standards for donor stool mass, final preparation volume, manufacturing technique or equipment. Commercial systems that can be carefully disinfected between uses is highly desirable for infection control, but they are associated with substantial costs and are therefore used inconsistently. Disposable equipment may be one alternative to decrease manufacturing costs. ${ }^{4,22}$ Although some of our programs performed validation studies to support their manufacturing processes and storage conditions, no industry standard exists regarding metrics for validation studies.

National surveys of FMT practices in the United Kingdom and in Ireland focused primarily on identifying hospitals where FMT is offered and analyzing barriers to FMT uptake; protocols for manufacturing and administration were not detailed. ${ }^{23-25}$ These studies showed that FMT programs are greatly affected by multiple factors including costs, local administrative pressures and availability of screening tests. Other investigators have reported on the development of single-site FMT programs, ${ }^{26,27}$ but, to our knowledge, a larger-scale comparison of protocols and practices between FMT programs has not been performed.

\section{Limitations}

Our study has limitations. The study sites may not represent all sites where FMT is performed within our region. We surveyed a convenience sample of members of an established network, which may have resulted in sampling bias; this would limit the generalizability of our results. In addition, since the study investigators were also survey respondents, our interpretations of the study findings may have been influenced by our own experiences and opinions. Two of the surveyed sites were in the process of developing FMT programs, and survey response may not represent their final approaches. Finally, we aimed to simply describe the FMT programs and did not collect data on the clinical outcome of FMT recipients, which would be important for guiding future recommendations for FMT programs.

\section{Conclusion}

We identified considerable diversity in FMT practices in southern Ontario. The 2 largest FMT program barriers in the region are a dependency on physicians and donor screening requirements. In Ontario, the costs of FMT programs are currently borne by hospitals and research funding. In planning future FMT programs in Canada, until such things as manufactured biotherapeutics are available, opportunities for systems-wide efficiencies such as creating regional FMT centres should be explored. Such an approach would concentrate expertise in FMT, ensure consistency in practices, enable trace-back in the event of FMT-related adverse events and reduce the need for numerous institutions to purchase costly donor screening tests and equipment.

\section{References}

1. van Nood E, Vrieze A, Nieuwdorp M, et al. Duodenal infusion of donor feces for recurrent Clostridium difficile. N Engl 7 Med 2013;368:407-15.

2. Cammarota G, Masucci L, Ianiro G, et al. Randomized clinical trial: faecal microbiota transplantation by colonoscopy vs vancomycin for the treatment of recurrent Clostridium difficile infection. Aliment Pharmacol Ther 2015;41: 835-43.

3. Youngster I, Sauk J, Pindar C, et al. Fecal microbiota transplant for relapsing Clostridium difficile infection using a frozen inoculum from unrelated donors: a randomized, open-label, controlled pilot study. Clin Infect Dis 2014;58:1515-22.

4. Lee CH, Steiner T, Petrof EO, et al. Frozen vs fresh fecal microbiota transplantation and clinical resolution of diarrhea in patients with recurrent Clostridium difficile infection: a randomized clinical trial. FAMA 2016;315:142-9.

5. Kelly CR, Khoruts A, Staley C, et al. Effect of fecal microbiota transplantation on recurrence in multiply recurrent Clostridium difficile infection: a randomized trial. Ann Intern Med 2016;165:609-16.

6. Hota SS, Sales V, Tomlinson G, et al. Oral vancomycin followed by fecal transplantation versus tapering oral vancomycin treatment for recurrent Clostridium difficile infection: an open-label, randomized controlled trial. Clin Infect Dis 2017;64:265-71.

7. Kao D, Roach B, Silva M, et al. Effect of oral capsule- vs colonoscopydelivered fecal microbiota transplantation on recurrent Clostridium difficile infection: a randomized clinical trial. FAMA 2017;318:1985-93.

8. Hota SS, Tomlinson G, Poutanen SM. Reply to Galperine et al and Jensen. Clin Infect Dis 2017;64:1293-5.

9. Kassam Z, Lee CH, Hunt RH. Review of the emerging treatment of Clostridium difficile infection with fecal microbiota transplantation and insights into future challenges. Clin Lab Med 2014;34:787-98.

10. Guidance document: Fecal microbiota therapy used in the treatment of Clostridium difficile infection not responsive to conventional therapies. Ottawa: Health Canada; 2015 (modified 2016 Oct. 18). Available: www.hc-sc.gc.ca/dhp-mps/consultation/ biolog/fecal_microbiota-bacterio_fecale-eng.php (accessed 2016 May 6).

11. Bakken JS, Borody T, Brandt LJ, et al.; Fecal Microbiota Transplantation Workgroup. Treating recurrent Clostridium difficile infection with fecal microbiota transplantation. Clin Gastroenterol Hepatol 2011;9:1044-9.

12. Cammarota G, Ianiro G, Tilg H, et al.; European FMT Working Group. European consensus conference on faecal microbiota transplantation in clinical practice. Gut 2017;66:569-80.

13. Census profile, 2016 census. Ontario [province] and Canada [country] (table). Ottawa: Statistics Canada; 2017. Available: http://www12.statcan.gc.ca/census -recensement/2016/dp-pd/prof/details/page.cfm?Lang=E\&Geo1=PR\&Code1= $35 \&$ Geo $2=$ PR \&Code2 $=01 \&$ Data $=$ Count $\&$ Search Text $=$ ontario $\&$ Search Type $=$ Begins \&SearchPR=01\&B1=All\&TABID=1 (accessed 2018 Feb. 8).

14. Kassam Z, Lee CH, Yuan Y, et al. Fecal microbiota transplantation for Clostridium difficile infection: systematic review and meta-analysis. Am 7 Gastroenterol 2013;108:500-8.

15. Guo B, Harstall C, Louie T, et al. Systematic review: faecal transplantation for the treatment of Clostridium difficile-associated disease. Aliment Pharmacol Ther 2012;35:865-75.

16. Gough E, Shaikh H, Manges AR. Systematic review of intestinal microbiota transplantation (fecal bacteriotherapy) for recurrent Clostridium difficile infection. Clin Infect Dis 2011;53:994-1002.

17. Cammarota G, Ianiro G, Gasbarrini A. Fecal microbiota transplantation for the treatment of Clostridium difficile infection: a systematic review. 7 Clin Gastroenterol 2014;48:693-702.

18. Drekonja D, Reich J, Gezahegn S, et al. Fecal microbiota transplantation for Clostridium difficile infection: a systematic review. Ann Intern Med 2015;162: 630-8.

19. Hamilton MJ, Weingardent AR, Sadowsky MJ, et al. Standardized frozen preparation for transplantation of fecal microbiota for recurrent Clostridium difficile infection. Am $\mathcal{F}$ Gastroenterol 2012;107:761-7. 
20. Tang G, Yin W, Liu W. Is frozen fecal microbiota transplantation as effective as fresh fecal microbiota transplantation in patients with recurrent or refractory Clostridium difficile infection: a meta-analysis? Diagn Microbiol Infect Dis 2017; 88:322-9.

21. Terveer EM, van Beurden YH, Goorhuis A, et al. How to: Establish and run a stool bank. Clin Microbiol Infect 2017;23:924-30.

22. Perez E, Lee CH, Petrof EO. A practical method for preparation of fecal microbiota transplantation. Methods Mol Biol 2016;1476:259-67.

23. Porter RJ, Fogg C. Faecal microbiota transplantation for Clostridium difficile infection in the United Kingdom. Clin Microbiol Infect 2015;21:578-82.

24. Prior AR, Kevans D, McDowell L, et al. Treatment of Clostridium difficile infection: a national survey of clinician recommendations and the use of faecal microbiota transplantation. 7 Hosp Infect 2017;95:438-41.

25. Quraishi MN, Segal J, Mullish B, et al. National survey of practice of faecal microbiota transplantation for Clostridium difficile infection in the UK. 7 Hosp Infect 2017;95:444-5.

26. Costello SP, Tucker EC, La Brooy J, et al. Establishing a fecal microbiota transplantation service for the treatment of Clostridium difficile infection. Clin Infect Dis 2016;62:908-14.

27. Tauxe WM, Dhere T, Ward A, et al. Fecal microbiota transplant protocol for Clostridium difficile infection. Lab Med 2015;46:e19-23.

Competing interests: Susy Hota has received a grant and honoraria for serving as a consultant and on the advisory board of Cubist (Merck) Pharmaceuticals. Susan Poutanen has received honoraria related to advisory boards from Merck, Paladin Labs and Accelerate Diagnostics, honoraria from Merck related to talks, and research support from Bio-Rad Laboratories, bioMérieux and Accelerate Diagnostics. Christine Lee is a member of advisory boards for Rebiotix and Summit Therapeutics and has received grants from Merck, Actelion Pharmaceuticals and Rebiotix for clinical research. Elaine Petrof is a cofounder and serves on the Scientific Advisory Board of NuBiyota. Jeff Powis has received a grant from Gilead. Daniel Ricciuto has received a grant from Astellas. Michael Silverman has received speaker honoraria from Merck. No other competing interests were declared.

Affiliations: Department of Infection Prevention and Control (Hota), University Health Network; Department of Medicine (Hota, Coburn),
University of Toronto; Department of Microbiology (Surangiwala, Paterson, Poutanen), University Health Network/Sinai Health System; Toronto General Hospital Research Institute (Coburn); Department of Laboratory Medicine and Pathobiology (Poutanen), University of Toronto, Toronto, Ont.

Contributors: Susy Hota and Susan Poutanen co-led the design of the study and the development of the survey. Susy Hota drafted the manuscript. Salman Surangiwala administered the survey and completed the initial data analysis. Aimee Paterson performed additional data analysis. Bryan Coburn reviewed and approved the survey before administration. All Southern Ontario Fecal Microbiota Transplantation (SOFT) Movement leads reviewed the study data for integrity, provided feedback on results, contributed to manuscript preparation and approved the final draft. All of the authors gave final approval of the version to be published and agreed to be accountable for all aspects of the work.

Southern Ontario Fecal Microbiota Transplantation (SOFT) Movement physician leads: Bryan Coburn, University Health Network, Toronto; Nick Daneman, Sunnybrook Health Sciences Centre, Toronto; Mark Downing, St. Joseph's Health Centre, Toronto; Christopher Graham, Trillium Health Partners, Mississauga; Susy S. Hota, University Health Network, Toronto; Jennie Johnstone, Public Health Ontario, St. Joseph's Health Centre, Toronto; Christine Lee, St. Joseph's Healthcare, Hamilton; Janine McCready, Michael Garron Hospital, Toronto; Elaine O. Petrof, Kingston General Hospital, Kingston; Susan M. Poutanen, Sinai Health Centre, University Health Network, Toronto; Jeff Powis, Michael Garron Hospital, Toronto; Daniel Ricciuto, Lakeridge Health, Oshawa; and Michael Silverman, St. Joseph's Hospital, London.

Funding: This work was supported by an Integrating Challenge Grant from the Department of Medicine, and the Department of Laboratory Medicine and Pathobiology, University of Toronto.

Supplemental information: For reviewer comments and the original submission of this manuscript, please see www.cmajopen.ca/content/6/2/ E184/suppl/DC1. 